\title{
Using Bookmark Visualizations for Self-reflection and Navigation
}

\section{Pooja Mathur}

University of Illinois at Urbana-

Champaign, Department of

Computer Science

201 N. Goodwin

Urbana, IL 61801 USA

pmathur2@illinois.edu

Karrie Karahalios

University of Illinois at Urbana-

Champaign, Department of

Computer Science

201 N. Goodwin

Urbana, IL 61801 USA

kkarahal@illinois.edu
Copyright is held by the author/owner(s).

CHI 2009, April 4 - 9, 2009, Boston, MA, USA

ACM 978-1-60558-246-7/09/04.

\begin{abstract}
Web users have been employing numerous methods for recalling websites. Bookmarks have been around for some time, but the usefulness of such a system has been under question. The lack of intuitive organization in web browsers forces users to make arbitrary choices on where to store bookmarks. As a result, bookmarks are often lost, never to be used again. These web pages a user bookmarks can say a lot about the user, though. A person's personality is reflected by the web pages a user visits and deems important enough to bookmark. By taking the user's bookmarks along with one's browser history and visualizing them, the user is able to notice things about oneself that he or she did not notice before. In this paper, we describe the iteration of a tool that visualizes all of user's bookmarks. We conclude with areas for future work.
\end{abstract}

\section{Keywords}

Bookmarks, visualizations, self-reflection, information organization

\section{ACM Classification Keywords}

H5.2. Information Interfaces and Presentation: User Interfaces - User-centered Design. H5.4. Information Interfaces and Presentation: Hypertext/Hypermedia Navigation. 


\section{Introduction}

The majority of web page visits are actually web page revisits. In 1995 a study [8] found that $58 \%$ of web pages visited by a user were pages the user had already seen. In 2000 another study [3] found that this number had increased to $81 \%$. However, very few people use bookmarks when revisiting these pages [8] There have been studies to investigate how people organize their bookmarks. Abrams et al. [1] found out that $37 \%$ of users do not organize their bookmarks at all. Without organization, bookmarks are easily lost or forgotten.

People can learn about themselves through their digital content. Goffman studied the "expressive component" [4] of social interaction. He found that the "expressive component" is how people create impressions of others and themselves. These impressions are considered the sources of "unapparent facts". In other words, people are able to learn things about others by observing different characteristics and elements. For example the clothes one wears and the things one does all say something about a person [4, 7]. In this case, we are looking at how one may learn about oneself through the expressive component of one's bookmarks and web history.

Viegas et al. created a tool to visualize a user's email data in 2004 for study. This tool, called PostHistory [9], took a user's emails and contacts and allowed the user to view how contacts became more and less prominent in one's life over time. The user could see and recall events in one's life that caused changes in one's email contacts. A new group project in school, joining a club or organization, these events lead to changes in email contacts. All this information was technically available to the users, but it was the visualizations that brought it to light.

\section{Related Work}

There have been many tools created for working with bookmarks. Kaasten and Greenberg [6] take a user's history, bookmarks, and back button and intertwine them into a tool to help users navigate pages they have previously visited. In this tool, a user navigates around visited web pages with more information. Users get an idea of how often they visit a page and whether or not it is bookmarked. In another tool, Hascoet also integrates bookmarks and history in a tool called BookMap [5]. BookMap gives users the ability to view their bookmarks in a radial tree. The nodes of the tree are thumbnails of web pages. Users are free to explore pages from here.

Another project studied how one person could learn about another using the other's web history [2]. The web pages that were used from the history were the most visited pages. People were able to learn that they had things in common with colleagues that normally did not come up in conversation at work. This project just offers a glimpse at the power web history holds.

\section{Bookmark Tool}

The tool we have created is an extension for Mozilla Firefox. It takes a user's bookmarks and displays them in a few ways. Each different view is supposed to give users the opportunity to see, notice and learn different things.

Our initial designs for the tool were very simple. They used minimal colors and did not portray how a user would navigate the system very well. However, the 


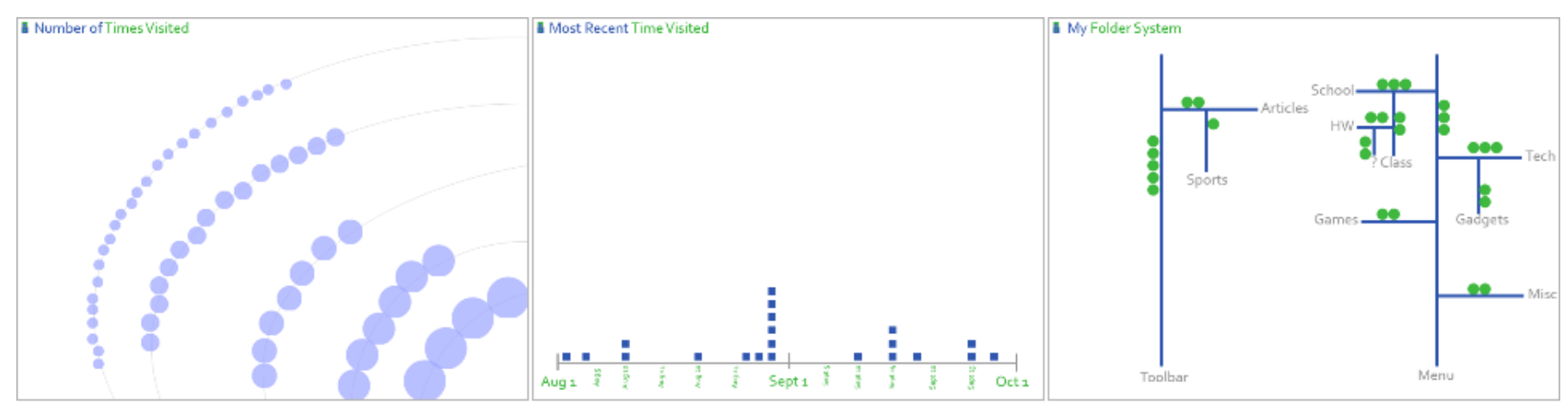

Figure 1. From left to right, one can see the initial mock-ups of the arcs, timeline, and tree visualizations. Some of the issues here were the lack of color and lack of scalability.

basic overall ideas remain. Bookmarks are represented as shapes in each of the visualizations. Figure 1 contains the initial mock-up designs for each of the visualizations. Originally we were only using blues and greens with grays as accents in the visualizations When this idea was presented to others, people stated that they felt overwhelmed by the lack of differentiation. Users did not know where to start because everything looked the same. This is when we started to introduce more color and gave colors meaning in the visualizations.

The shapes have colors and the colors categorize the bookmarks in the current implementation of the tool. The default categories are set to gather information from the domain name. If the domain ends in ".edu" for example, the bookmark shape is colored green. For all of the default categories, see Figure 2. When the user mouses-over a bookmark, more information is shown, such as the bookmark's title, the number of times the user has visited that bookmark and the last date the user visited that bookmark. An example of the information caption box is found in Figure 3a. The user can click on any of these bookmark shapes on any of the visualizations and a new tab opens up displaying that web page.

\section{Arcs View}

The first visualization places the bookmarks on a set of arcs. This view is intended to give the user an idea of which bookmarks are used the most and which bookmarks get little to no attention. The bookmarks are placed in order of visit frequency and in case of ties alphabetical order. Then the shapes are distributed among the five arcs on the screen. The more often a bookmark is visited, the larger the shape representing the bookmark will be. These larger shapes appear on the shortest arc near the bottom of the screen and the least visited, smaller bookmark shapes appear on the arc closest to the top of the screen. Figure $3 a$ has an image of the current implementation of the arcs view. The arcs were moved to the bottom-center to allow more bookmarks to appear on an arc because of the longer arcs lengths it affords.

By easily seeing that there are pages that are not visited often, the user has the opportunity to explore these forgotten sites or decide that one needs to clean 


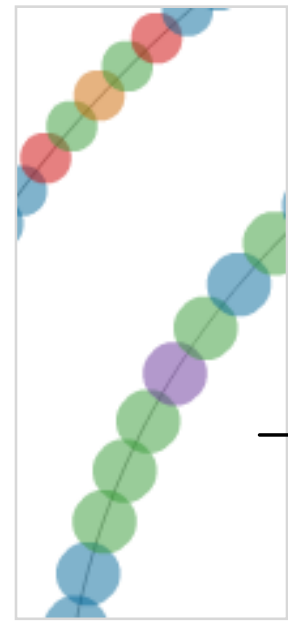

Figure 3b. This is the actual size of the shapes in this image of the arcs view.

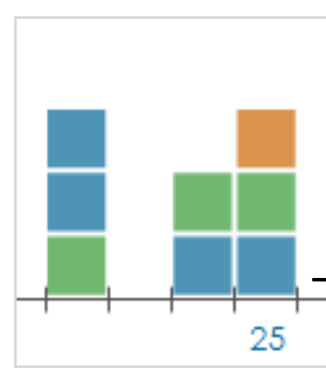

Figure 4b. This is the actual size of the shapes in the timeline view. out his or her bookmarks folder. Users can also use the most visited websites to see what pages one feels are most important.

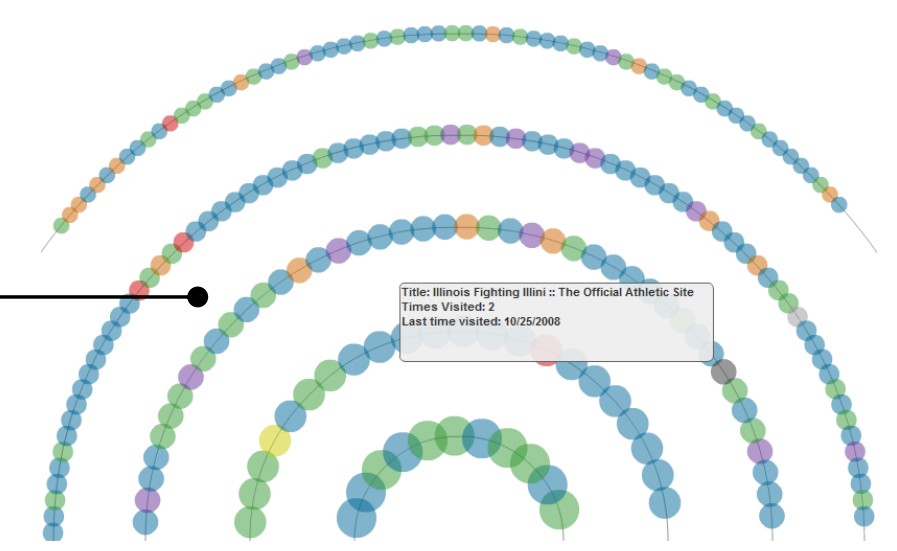

Figure 3a. This is an image of the arcs view for a user with $\sim 200$ bookmarks. One can also see a sample caption box.

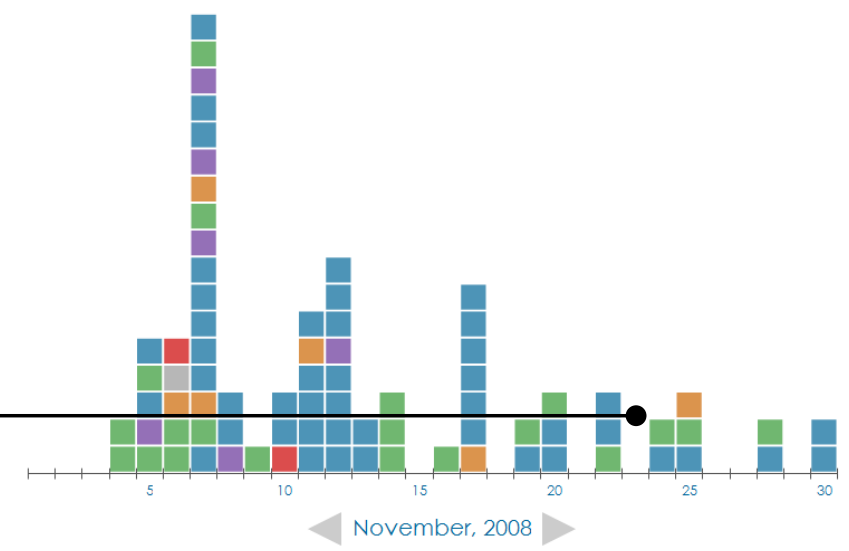

Figure 4a. This is an example of part of the timeline view for a user in the month of November 2008.
Timeline View

In this view, one's bookmarks are placed on a timeline based on the last time a user has visited a bookmark. In this visualization, bookmarks are represented as squares. When first navigating to this view, the most recent month with bookmark activity is shown. Users employ the arrows near the bottom of the screen to navigate to other months with bookmark activity. If there is no other activity, the arrows are disabled.

With this view, users visit previous months of bookmark activity to see what bookmarks they have not visited in a long time. By doing this, users have the opportunity to remember past events. For example, a set of bookmarks were created for a project and once the project was completed, the bookmarks were no longer used. The timeline view can not only point out that those bookmarks have not been in use for some time, but it allows the user to recall memories from that project.

Just like PostHistory [9], users have the opportunity to use their personal digital content to for self-reflection and memory recollection. It is like the bookmarks are virtual souvenirs of one's life. In Figure 4a, one can see that there were only a few bookmarks visited during the end of November. By seeing the bookmarks were rarely used at this time, the user might question the decrease in activity. Then the user may notice that this happens to coincide with this user's Fall Break schedule.

Tree View

This last visualization offers users the opportunity to learn about their organization skills. Each bookmark is represented by a circle, and the folders are represented 
by squares with rounded corners. Users mouse over and click the bookmarks just like in the other visualizations. The folders, however, cannot be clicked. Users can also view all three of their folder systems separately. That is, Firefox has three bookmark folder systems. There is the bookmarks menu, toolbar bookmarks, and the unfiled bookmarks. By using the arrows at the bottom of the screen, the user navigates between these three folder systems. Figure $5 a$ shows the bookmarks menu tree for a user with numerous bookmarks and folders. Figure $5 \mathrm{~b}$ shows the actual size of the folder and shapes in the visualization.

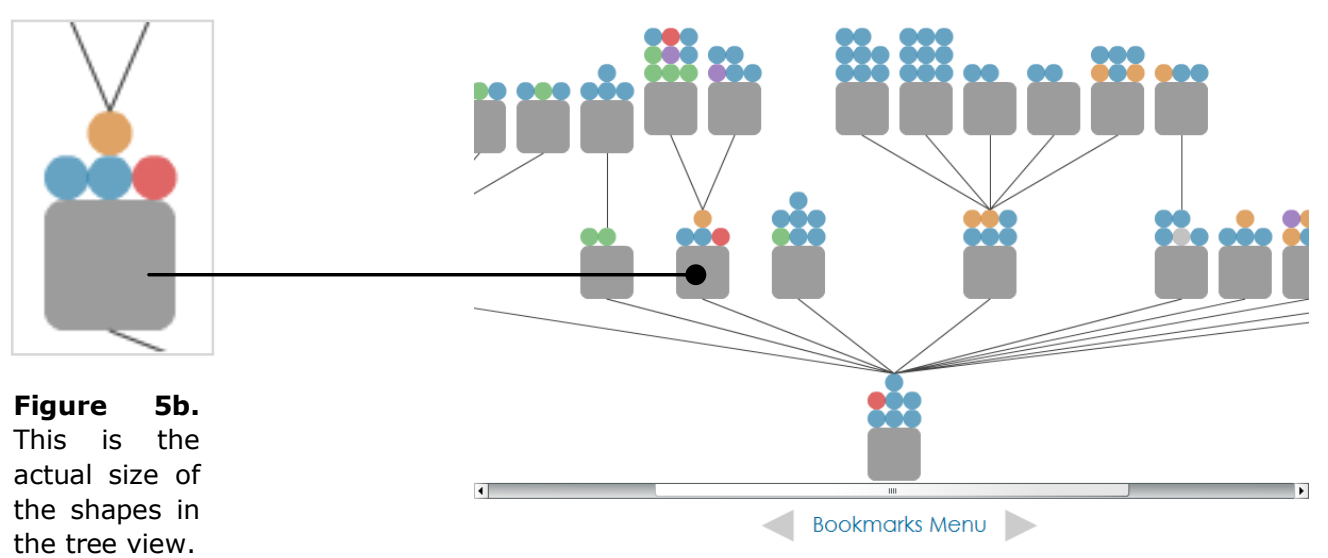

Figure 5a. This is an example of the tree view for a user with an extensive folder system. Arrows are also available here to view the other folder systems.

This visualization changed the most from the original design in the mock-ups (see Figure 1 - right). The biggest issue here was that the visualization could only handle a certain number of sub folders before it ran out of screen real estate. We had to come up with a design that was not limited to the screen. In the end we chose a more traditional view of a folder hierarchy displayed as a tree (see Figure 5a). Scrollbars are enabled for both the horizontal axis and vertical axis, if the number of folders and bookmarks call for it so the size of the tree is not sacrificed.

\section{Customization}

The user can also customize the colors of bookmarks and categories a color represents in the visualizations. This customization tool allows the user to change the label for 6 of the 7 colors (blue is always "Default"). After making one's own categories, the user can assign those categories to one's own bookmarks.

\section{Future Work}

There is still much work to be done on this tool. Many people who have looked at the visualizations have stated that arcs visualization is not as intuitive to understand. One suggestion is to make the size of the bookmark shape directly proportional to the number of visits. Another suggestion is to make the secondary ordering parameter the date the web page was bookmarked. The bookmarks would still be first ordered by visit frequency, but then second by recency of addition.

There are also many opportunities to use a similar tool for other types of personal digital content. We initially created this tool to use with a person's web browser history and bookmarks. With this tool, users can revisit old forgotten web pages, recall memories about why they bookmarked the pages in the first place, and learn about themselves.

If one were to think for a moment, there are many types of personal digital content. For this example, 
however, we look at digital pictures. Think about the large number of pictures users have in their photo collections. Digital cameras and larger hard drives have enabled people to create gigantic photo collections. People have so many pictures that most of them sit in folders untouched. They are rarely viewed, if ever.

If there was a tool similar to the bookmark tool we have created, users would get the opportunity to view and explore their photo collections. A timeline view of one's photo collection could show the user when photos were taken. For example, the user takes a trip to New Zealand for a vacation in January of 2008. That timeline may have hundreds of squares in the timeline visualization from January 2008, but no photos around it. Going back farther, the user may have photos from a day at an amusement park or a family reunion. The next visualization might organize photos by tags or titles. A third visualization would be similar to the tree view, showing the user how they organize their photos. A visualization similar to the arcs view could show the user which photos they never see.

Home videos would also benefit from a similar tool. A baby's first steps, a graduation, a wedding, all of these videos may just sit on a hard drive. Visualizations to remind the user that these videos existed would lead users to get more out of the content that they themselves created.

No one doubts that photos and videos are catalysts for recalling memories. By exploring the collections in a new way, these pieces of personal content would not be forgotten.

\section{Acknowledgements}

We would like to thank Kelsey Schlarman, without whom this tool would not have been completed. We would also like to thank everyone in CS465 at UIUC for their feedback on our project.

\section{References}

[1] D. Abrams, R. Baecker, and M. Chignell. Information archiving with bookmarks: Personal web space construction and organization. Proc. CHI 1998, ACM Press (1998), 41-48.

[2] M. Bilezikjian, J. C. Tang, J. Begole, and N. Yankelovich. Exploring web browser history comparisons. Proc. CHI 2002, ACM Press (2002), 828829.

[3] A. Cockburn and B. McKenzie. What do web users do? An empirical analysis of web use. Int. J. HumanComputer Studies, (2000).

[4] E. Goffman. The Presentation of Self in Everyday Life. Doubleday, New York, 1959.

[5] M. Hascoet. A user interface combining navigation aids. Proc. Hypertext 2000. ACM Press (2000), 224225.

[6] S. Kaasten and S. Greenberg. Integrating back, history and bookmarks in web browsers. Proc. CHI 2001. ACM Press (2001), 379-380.

[7] D. Norman. Emotional Design: Why we love (or hate) everyday things. Basic Books, New York, 2005.

[8] L. Tauscher and S. Greenberg. How people revisit web pages: empirical findings and implications for the design of history systems. Int. J. Human-Computer Studies, (1997), 97-137.

[9] F. B. Viegas, d. boyd, D. H. Nyugen, J. Potter, and J. Donath. Digital artifacts for remembering and storytelling: PostHistory and social network fragments. Proc. HICSS 2004. IEEE Computer Society (2004). 